\title{
Preface
}

Life persists not in three but in four dimensions - a persistence, which is above all dependent upon the maintenance of order: order within the cell, order within the organism, and order in the relationship of the organism to the world outside it. (Rose, 1999: 878)

This book has two important aims. First, to help other educators understand the journeys other colleagues have undertaken to become entrepreneurship educators. Second, and more importantly I would argue, to introduce and establish order in the domain of enterprise and entrepreneurship education. Order that I believe holds the power to propel our domain of education onwards to new heights. This book has been crafted to achieve both aims, and the concluding comments at the end of each section are directed at the order that has been achieved in this book through the attempted use of a common language.

The context within which this books aims to achieve these two outcomes is however very dynamic and difficult to discern. On one hand we have the ever-increasing interest and provision of enterprise and entrepreneurship education (hereinafter EE) globally. The world is awash with EE, it is found in kindergartens all the way through to universities and beyond, and it is also increasingly found in all discipline areas of learning. EE is offered, as a solution to those displaced from the jobs market, be they aged, returning from defending their country or just looking to change lanes. In anticipation of the significant and enduring contribution EE will make to personal development and more broadly, societal evolution, highly integrated entrepreneurial ecosystems are forming, sponsored by numerous interests. In this sense, EE is a game within many other games. The rules we may choose individually or as a domain are subject to the acceptance of the various hosts who accommodate EE.

On the other hand, especially in the context of Higher Education (HE), other forces are at work. Where one might see centrifugal forces at play in the global expansion of EE, it would seem that various centripetal forces are acting in an opposite direction upon educational landscape more generally. While it is not uncommon to view centrifugal and centripetal forces acting simultaneously, the relative power of centripetal forces must counterbalance centrifugal forces 
for the status quo to remain. The view of many pundits ${ }^{1}$ is that, overall, the centripetal forces presently have the upper hand.

Drawing on the logic expressed by Steven Rose (in the opening statement of this chapter), in order to persist, EE (in its localised contexts) will be dependent upon order: order within the curriculum, order within the institution, and order in its relationship to the outside world. The teaching practices and content/ process focus within a curriculum do not insulate EE against the vagaries of the future. Any host institution of EE is not immune to shocks from its external environment and must be able to adapt to less than optimal conditions and/or exploit external opportunities.

The chapters in this book enable you to see the emerging EE lifeline that each educator is developing in their respective contexts, vis-à-vis their practice and its contribution to an EE related programme. What is very clear from each contributor is the variety of different motivations, approaches, contexts and challenges they have all faced so far in their journeys. This book has been designed to amplify such differences, doing so through the use of a common language. This common language can be harnessed through the use of activity theory. ${ }^{2}$

\section{ACTIVITY THEORY}

Activity theory can be used as a lens to view all forms of EE, regardless of differences in student activity, institutional approach and/or educator and community factors. Commonly referred to as cultural-historical activity theory (CHAT) ${ }^{3}$ activity theory enables one to view and understand the interaction between human actors and their actions, as they occur within culturally produced systems where specific goals related to identifiable outcomes are pursued.

As I have noted elsewhere ${ }^{4}$ there are five key principles that control the use of activity theory. ${ }^{5}$

First, the activity setting is the primary unit of analysis. Second, multiple perspectives are used to ensure multiple voices are used to identify contradictions in the system through which transformation and innovative change is possible. Third, historicity is used to ensure prior change in the system is fully understood. Fourth, contradictions are sought within the activity system so that any identified system transformations could eliminate inherent tensions. Fifth, by embracing identified contradictions we can productively contemplate the nature of change required to improve the activity system. Therefore, activity theory provides a means for EE to be viewed as an activity system where improvements, large or small, are always possible. 
In the context of this book, activity theory is introduced to provide an additional preliminary role to any consideration of system improvement. Here, the role is to establish a lens through which the contributions of each educator can be contemplated in an interconnected manner. The chapters of Elinor Vettraino and Alex Maritz are standout examples of how EE practice can be discussed using the activity theory lens.

In Figure I.1, the six elements of any activity system are highlighted. First we have the subjects: people such as students, educators and/or administrators. Second, we have tools, often in our context, a curriculum. Third, there are rules, which govern the actions and influence the design of all other elements. Fourth, we have roles, which are aligned to the expected behaviour of the subjects and other related stakeholders. Fifth, we have the institutional and/or community structures and related culture which directly influence the relationships that exist within the activities. Finally, there are the intended outcomes intended within the activity system.

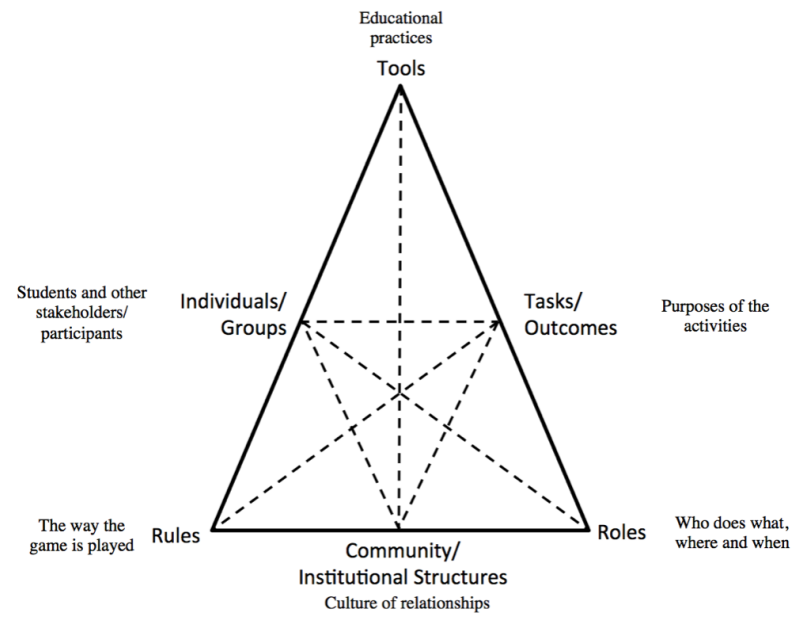

Figure I.1 Activity system model (adapted from Engeström, 1999)

It is my hope that activity theory can provide access to a common language (i.e. rules, roles, community, subjects, outcomes and tools) that will accommodate a wide variance of voices, whose stories comprise the chapters that make up this book. In maintaining the individuality of each contributor's voice and their unique contexts, I hope, that as you 'step in and out' of each context, the common lens used to present each chapter will increase your comprehension, despite your potential unfamiliarity with some of the contexts. The logic 
behind this design is as follows. Each reader of this book will most likely not have specialised experience across all four of the distinct areas (i.e. the early years, the pre-graduate years, the graduate years and the post-graduate years). Yet, I believe that there is much to be gained from understanding the practice of educators operating outside of our natural areas of operation. Therefore, I encourage you to allow the thinking of all of the contributors to wash over you, to stimulate and challenge your thinking. Most of all, I hope you take up the challenge at the conclusion of the book to explore the development of your scholarship of teaching and learning in the context of EE, becoming a philosopher of EE along the way!

\section{NOTES}

1. See the various works of Carey (2015), McGee (2015), Soliday and Lombardi (2018) and Staley (2019) to access a good cross-section of the concerns for those trends that are shaping the educational landscape.

2. See Engeström $(1987,2001)$ to gain a broad overview of the foundational ideas of activity theory and its development over time, and Barnes (2012) for a more recent example of its use to improve educational systems.

3. An excellent overview of the use of activity theory in the education domain is provided by Bligh and Flood (2017).

4. See Jones (2019a: 21).

5. See Barnes (2012). 
Colin Jones - 9781789900033 Downloaded from PubFactory at 04/26/2023 01:15: 05PM via free access 